\title{
Solution of Low-Frequency Electromagnetics Problems Using Hierarchical Matrices
}

\author{
Mahdi Kazempour ${ }^{1,2}$ and Levent Gürel ${ }^{1,2}$ \\ ${ }^{1}$ Department of Electrical and Electronics Engineering, Bilkent University, Ankara, TR-06800, Turkey \\ ${ }^{2}$ Computational Electromagnetics Research Center (BiLCEM), Ankara, TR-06800, Turkey
}

kazempour@ee.bilkent.edu.tr

\begin{abstract}
Fast and accurate solutions of low-frequency electromagnetics problems are obtained with an iterative solver based on hierarchical matrices. Iterative solvers require matrix-vector multiplications (MVMs). The results show significant reductions both in CPU time and memory consumption compared to the $\mathcal{O}\left(N^{2}\right)$ complexity of ordinary matrix filling and MVM.
\end{abstract}

\section{INTRODUCTION}

Surface integral equations (SIE) are commonly used to formulate scattering and radiation problems. Numerical solutions of integral equations require surface discretization that is achieved by the method of moments (MoM). MoM discretization of SIE yields a dense $N \times N$ matrix equation. Many methods have been proposed to accelerate the solutions of matrix equations. Most of these so-called "fast" methods take advantage of redundancies in the MoM impedance matrix.

One of the most powerful methods is the multilevel fast multipole algorithm (MLFMA). Due to its reduced computational complexity of $\mathcal{O}(N \log N)$, this method has been widely used in recent years to solve extremely large electromagnetics problems [1]. MLFMA is a kernel-dependent method that uses the properties of the Green's function.

Alternatively, there are also purely algebraic methods, which rely on some elements of the impedance matrix and are independent of the problem's kernel. A subset of these methods is composed of solvers based on hierarchical matrices $(\mathcal{H}$ matrices) [2].

MLFMA suffers from a low-frequency breakdown that effectively limits the minimum size of the lowest-level clusters to approximately one-fourth of a wavelength $(\lambda / 4)$. Many techniques are provided in the literature to overcome the lowfrequency breakdown of MLFMA, but all of them require major modifications to the implemented codes. [3].

Solvers based on $\mathcal{H}$-matrices do not suffer from the above problem due to their purely algebraic nature. This paper presents a brief description of $\mathcal{H}$-matrices as well as numerical results for low-frequency simulations.

\section{IMPLEMENTATION DETAILS}

Discretizing a SIE with MoM using the Rao-Wilton-Glisson (RWG) [4] basis and testing functions and the Galerkin scheme results in a system of linear equations

$$
\overline{\boldsymbol{Z}}_{(N \times N)} \cdot \boldsymbol{x}_{(N \times 1)}=\boldsymbol{b}_{(N \times 1)},
$$

where $\bar{Z} \in \mathbb{C}^{N \times N}$ is a dense impedance matrix, $\boldsymbol{x} \in \mathbb{C}^{N \times 1}$ is the vector of unknown coefficients, $\boldsymbol{b} \in \mathbb{C}^{N \times 1}$ represents the excitation with a plane wave, and $N$ is the number of unknowns [1]. We denote the index set of all basis (and testing) functions by $\mathcal{I}=\{1,2, \cdots, N\}$. Consider two subsets $\tau$ and $\sigma$ of $\mathcal{I}$. Interactions between the unknowns belonging to the sets $\tau$ and $\sigma$ generate the subblock $\overline{\boldsymbol{Z}}^{\text {sub }}$ of $\overline{\boldsymbol{Z}}$, and the size is $|\tau| \times|\sigma|$, where $|\cdot|$ denotes the cardinality of a set. The subblock $\bar{Z}^{\text {sub }}$ is called admissible if both of the following conditions are satisfied:

$$
\min \{|\tau|,|\sigma|\} \geq n_{\min }
$$

and

$$
\min \left\{\operatorname{diam}\left(\Omega_{\tau}\right), \operatorname{diam}\left(\Omega_{\sigma}\right)\right\} \leq \operatorname{dist}\left(\Omega_{\tau}, \Omega_{\sigma}\right),
$$

where $\Omega_{\tau}$ and $\Omega_{\sigma}$ are domains belonging to subsets $\tau$ and $\sigma$, respectively, and $n_{\text {min }}$ denotes the minimum cluster size belonging to an admissible block. An $\mathcal{H}$-matrix representation of $\bar{Z}$ is associated with the admissibility condition. In this representation, an inadmissible block keeps its original fullmatrix representation, while an admissible block is factorized into a low-rank form. In other words, each admissible block $\overline{\boldsymbol{Z}}^{\mathrm{adm}}$ can be written in the form of $\overline{\boldsymbol{Z}}_{(m \times n)}^{\mathrm{adm}} \approx \tilde{\boldsymbol{Z}}_{(m \times n)}=$ $\overline{\boldsymbol{U}}_{(m \times k)} \cdot \overline{\boldsymbol{V}}_{(k \times n)}$, where $k \in \mathbb{N}$ is the effective rank of $\overline{\boldsymbol{Z}}_{(m \times n)}^{\mathrm{adm}}$ and $k<\min \{m, n\}$. The aim of this compression is to achieve

$$
\left\|\overline{\boldsymbol{Z}}_{(m \times n)}-\tilde{\boldsymbol{Z}}_{(m \times n)}\right\|_{F}^{2} \leq \epsilon\left\|\overline{\boldsymbol{Z}}_{(m \times n)}\right\|_{F}^{2},
$$

for a given (and controllable) $\epsilon$, where $\|\cdot\|_{F}^{2}$ denotes the Frobenius matrix norm. This way, the required storage is reduced from $m \times n$ to $k \times(m+n)$. The computational cost of a solver based on $\mathcal{H}$-matrices is related to calculations of the compressed format of admissible blocks.

According to conditions (2) and (3), once we construct a tree structure, we can partition the impedance matrix and create an $\mathcal{H}$-matrix. The aim is to compress and factorize an admissible block $\overline{\boldsymbol{Z}}_{(m \times n)}$ such that it can be expressed as the product of two matrices with a given accuracy $\epsilon$. Singularvalue decomposition (SVD) is an approved method to obtain a low-rank approximation, however, it has a high computational cost.

Instead, we use the adaptive cross approximation (ACA) algorithm [5] to obtain

$$
\overline{\boldsymbol{Z}}_{(m \times n)} \approx \overline{\boldsymbol{U}}_{\left(m \times k^{\prime}\right)} \cdot \overline{\boldsymbol{V}}_{\left(k^{\prime} \times n\right)}^{T},
$$


TABLE I

MEMORY AND TIMING RESUlTS

\begin{tabular}{|c|c|c|c|c|c|c|}
\hline $\mathrm{N}$ & $\begin{array}{c}\text { Memory } \\
(\%)\end{array}$ & level & $\begin{array}{c}\text { Fill } \\
(\mathrm{sec})\end{array}$ & $\begin{array}{c}\text { Solution } \\
(\mathrm{sec})\end{array}$ & $\begin{array}{c}\text { Frequency } \\
(\mathrm{MHz})\end{array}$ & $\begin{array}{c}\text { Mesh } \\
(\mathrm{cm})\end{array}$ \\
\hline 468 & 96.5 & 3 & 79 & 0.03 & 10 & 30 \\
\hline 810 & 88.7 & 3 & 226 & 0.11 & 15 & 20 \\
\hline 1566 & 65.4 & 4 & 847 & 0.4 & 20 & 15 \\
\hline 3690 & 40 & 4 & 3774 & 1.7 & 30 & 10 \\
\hline 6132 & 25.8 & 5 & 8503.7 & 3.2 & 40 & 7.5 \\
\hline 7992 & 21.4 & 5 & 15526 & 5.1 & 50 & 6.6 \\
\hline 13914 & 13.9 & 6 & 45027 & 14.1 & 60 & 5 \\
\hline
\end{tabular}

where $\overline{\boldsymbol{Z}}_{(m \times n)}, \overline{\boldsymbol{U}}$, and $\overline{\boldsymbol{V}}$ are complex matrices. Then, we employ a QR factorization for $\bar{U}$ and $\bar{V}$ to obtain

$$
\overline{\boldsymbol{Z}}_{(m \times n)} \approx \overline{\boldsymbol{Q}}_{U\left(m \times k^{\prime}\right)} \cdot \overline{\boldsymbol{R}}_{U\left(k^{\prime} \times k^{\prime}\right)} \cdot \overline{\boldsymbol{R}}_{V\left(k^{\prime} \times k^{\prime}\right)}^{T} \cdot \overline{\boldsymbol{Q}}_{V\left(k^{\prime} \times n\right)}^{T} \cdot
$$

We apply the reduced SVD (rSVD) to recompress the matrix

$$
\overline{\boldsymbol{S}}_{\left(k^{\prime} \times k^{\prime}\right)}=\overline{\boldsymbol{R}}_{U\left(k^{\prime} \times k^{\prime}\right)} \cdot \overline{\boldsymbol{R}}_{V\left(k^{\prime} \times k^{\prime}\right)}^{T},
$$

so that the final compressed format of $\overline{\boldsymbol{Z}}_{(m \times n)}$ becomes

$$
\overline{\boldsymbol{Z}}_{(m \times n)} \approx \overline{\boldsymbol{A}}_{(m \times k)} \cdot \overline{\boldsymbol{B}}_{(k \times n)}^{T},
$$

where $k$ denotes the minimal rank. The computational complexity of the provided compression method is less than SVD for each admissible block [2]. Figure 1(b) illustrates this fact. By this method, we can represent the matrix $\overline{\boldsymbol{Z}}_{(N \times N)}$ as a decomposition into full matrices ( $\boldsymbol{F}$-matrices) and compressed matrices $(\mathcal{R}(k)$-matrices).

Iterative solvers require at least one matrix-vector multiplication (MVM) in each iteration. Once we generate the $\boldsymbol{F}$-matrices and $\mathcal{R}(k)$-matrices, the MVM is computed in a block-wise manner. For those subblocks that are full matrices, the standard MVM is performed, while for the $\mathcal{R}(k)$ subblocks, this involves two steps. Note that the complexity of multiplying the $\mathcal{R}(k)$-matrix with a vector is the same as the complexity of its storage requirement, i.e., $k \times(m+n)$ [5].

\section{NUMERICAL RESUlts}

We demonstrate CPU time and memory usage of scattering problems that are formulated by combined-field integral equations (CFIE) with a combination coefficient of 0.5 and discretized with the RWG [4] functions on small planar triangles.

To examine the low-frequency solution of the proposed solver, the smallest box size is chosen to be $0.025 \lambda$ and the geometries are meshed with an average triangle size of $0.01 \lambda$. The geometry is a sphere with a radius of $1 \mathrm{~m}$. As these simulations are performed at various frequencies from $10 \mathrm{MHz}$ up to $60 \mathrm{MHz}$, the electrical size increases and the number of unknowns grows from 468 to 13,914 . Table I presents the results for the time and memory usage. Figure 1(a) reports the memory usage as the ratio of the used memory compared to MoM. Figure 1(b) shows that the computational complexity of the solver based on $\mathcal{H}$-matrices is lower than $\mathcal{O}\left(N^{2}\right)$ and that the proposed method can be used as a fast solver.

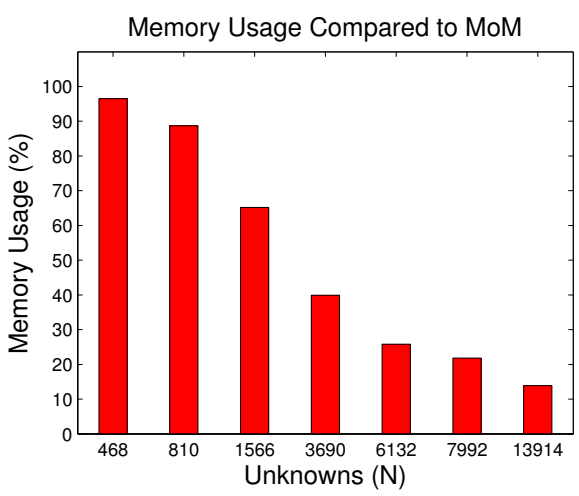

(a)

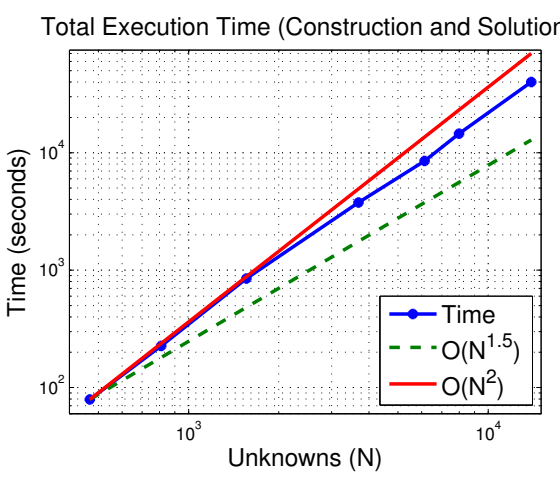

(b)

Fig. 1. Numerical results: (a) Ratio of the required memory in comparison to MoM. (b) CPU time together with $\mathcal{O}\left(N^{2}\right)$ and $\mathcal{O}\left(N^{1.5}\right)$ curves.

\section{CONCLUSIONS}

With the proposed solver, a significant reduction in memory (up to $86 \%$ ) and CPU time is achieved, compared to solvers based on MoM. Furthermore, the proposed solver takes advantage of its algebraic nature: distinct from kernel-dependent solvers, such as MLFMA, the solver based on $\mathcal{H}$-matrices is effectively applied on low-frequency scattering and radiation problems with no modifications needed.

\section{ACKNOWLEDGMENT}

This work was supported by the Scientific and Technical Research Council of Turkey (TUBITAK) under Research Grants 110E268 and 111E203, and by contracts from ASELSAN, Turkish Aerospace Industries (TAI), and the Undersecretariat for Defense Industries (SSM).

\section{REFERENCES}

[1] Ö. Ergül and L. Gürel, "Accurate solutions of extremely large integralequation problems in computational electromagnetics," Proc. IEEE vol. 101, no. 2, pp. 342-349, 2013.

[2] W. Chai and D. Jiao, " $H$ - and $H^{2}$-matrix-based fast integral-equation solvers for large-scale electromagnetic analysis," IET Microw. Antennas Propag., vol. 4, no. 10, pp. 1583-1596, 2010.

[3] H. Wallen and J. Sarvas, "Translation procedures for broadband MLFMA," Prog. Electromagn. Res., vol. 55, pp. 47-78, 2005.

[4] S. M. Rao, D. R. Wilton, and A. W. Glisson, "Electromagnetic scattering by surfaces of arbitrary shape," IEEE Trans. Antennas Propagat., vol. 30, pp. 409-418, 1982

[5] M. Bebendorf, Hierarchical Matrices. Berlin: Springer, 2008. 